\title{
Evaluation of Neurocognitive Abilities in Patients Undergoing Carotid Endarterectomy Surgery
}

\author{
Zafer Cengiz Er ${ }^{1}$, Ferit Çiçekçioğlu ${ }^{2}$, Kivanc Atilgan² \\ 'Department of Cardiovascular Surgery, Bozok University Research and Application Hospital, Yozgat, Turkey; \\ ${ }^{2}$ Department of Cardiovascular Surgery, Medicine Faculty, Bozok University, Yozgat, Turkey
}

\section{ABSTRACT}

Objective: To evaluate the differences in neurocognitive abilities between the preoperative and postoperative periods following carotid endarterectomy (CEA), due to carotid artery stenosis, and to evaluate the effectiveness of CEA on neurocognitive abilities in the future.

Material and methods: Thirty-eight cases of CEA surgery at Bozok University Faculty of Medicine Research Hospital between January 2015 and June 2020 were examined. Neurocognitive tests were performed on carotid endarterectomy patients one day before the operation and on the $2 \mathrm{nd}$, 4th, and 30th postoperative days. The effect of CEA on cognitive results has been investigated.

Results: Of the patients, eight were female (21.1\%), 30 were male $(78.9 \%)$, and the mean age was $66 \pm 4.09$. Thirtytwo $(84.21 \%)$ of the patients were operated on under general anesthesia and six (15.78\%) under regional anesthesia. A shunt was used in 19 patients. Right carotid endarterectomy was performed in 20 cases and left carotid endarterectomy in 18 cases. We used the primary closure technique in two of 38 cases and patches on 36 of them. We used Dacron in 21 cases, PTFE in 12 cases, and saphenous vein as a patch in three cases. In the WMS digit spam and recall scores, the postoperative period fell on the 2 nd day, and then on the 4th and 30th day after the operation, there was a low level of increase over time. Compared with the preoperative period, the learning score was found to be the lowest on the 2 nd day, lower on the 4th day compared with the preoperative period and improved compared with the preoperative period on the 30th day. There was no decrease in the verbal fluency test score results after the operation, on the contrary, it was observed minimally. The test score results cumulatively were decreased in the early postoperative periods compared with the preoperative period and increased on the 30th day compared with the preoperative period.

Conclusion: The purpose of CEA in the past was the prevention of ischemic stroke and cerebrovascular disease (CVD) rather than neurocognitive recovery. Factors affecting

Received October 7, 2020; received in revised form November 19, 2020; accepted November 23, 2020.

Correspondence: Kivanç Atılgan, MD, Bozok Üniversitesi Araştırma ve Uygulama Hastanesi Kalp ve Damar Cerrabisi Anabilim Dal, 66100 Yozgat, Turkey, Telephone+90354-2127060 (e-mail: kivancatilgan@gmail.com) neurocognition in CEA are multifactorial. Preservation and improvement of neurocognition are more important than any other period of history. By prioritizing cognitive abilities in the treatment of carotid stenosis, individualization of the treatment will help maximize the increase in cognitive abilities by providing optimum benefit to the patient of each factor.

\section{INTRODUCTION}

In many studies, carotid endarterectomy (CEA) has been shown to prevent the development of stroke [North American Symptomatic Carotid Endarterectomy Trial Collaborators 1991; European Carotid Surgery Trialists' Collaborative Group 1991]. Neurocognitive abilities are the mental processes of memory and perception that provide learning, problem-solving and future planning, and cognitive dysfunction is defined as a decrease in these processes [Hanning 2005]. The distinction between cognitive deficit and neurological deficiency is that the first is related to the loss of mental processes, such as the ability to think and remember, and the second is related to movement, sensory, or motor function loss. Vascular neurocognitive dysfunction (VNCD) due to vascular insufficiency is a clinical entity that causes cognitive impairment, and carotid stenosis plays an important role in its etiology [Bowler 2002]. Studies have shown that carotid artery stenosis leads to a decrease in neurocognitive abilities [Jackson 2015]. However, according to the research made on the effect of the treatment of stenosis on cognitive skills, there are controversial results and consensus has not been reached. There have been studies showing improvement in cognitive function, decreased function, mixed results, or no change [Feliziani 2010; Rasmussen 2006].

There are a variety of reasons that will affect cognitive abilities after CEA. These include microembolization, general anesthesia, use of carotid clamps, hypoxia during the operation, and hyperperfusion due to postoperative regulation disorder. The age of the population with CEA is advanced and probably has comorbidities that would be related to previous different degrees of cognitive impairment [Busse 2003]. The effects of the surgical process on existing comorbidities and the treatments applied may also be other factors on cognitive results. Our aim in this study is to compare the preoperative and postoperative neurocognitive states of patients with CEA and to examine how the elimination of carotid stenosis affects neurocognitive abilities. 


\section{MATERIAL AND METHODS}

Thirty-eight cases undergoing carotid endarterectomy surgery at Bozok University Medical Faculty Research Hospital between January 2015 and June 2020 were included in our prospective study. Eight of the patients (21.1\%) were female, 30 of the cases $(78.9 \%)$ were male and the mean age was $66 \pm$ 4.09. Since our cases were interned, additional diseases were investigated, and the pathologies detected were treated in the preoperative period. Thirty-two of the patients were operated on under general anesthesia and six under regional anesthesia. Right carotid endarterectomy was performed in 20 (52.63\%) and left carotid endarterectomy in 18 (47.36\%) of the cases. Cases with asymptomatic stenosis> $70 \%$ and symptomatic> $50 \%$ in angiography results were accepted as CEA operation indication. CEA performed with primary suturing and patching is called conventional (classical) endarterectomy in terminology. Our cases were operated with the classical technique by the same operational team. The cases had stopped smoking at least one week before the operation. In patients with hypertension and chronic obstructive pulmonary disease (COPD), preoperative blood pressure was regulated and the proper bronchodilator treatment of pulmonary diseases was provided. Blood glucose regulation of diabetic patients and antilipidemic treatments of patients with hyperlipemia were performed. In addition to blood sugar and cholesterol, vitamin B12, and vitamin D levels, which are effective factors in cognitive abilities, were examined, and replacement therapy was provided for those who were deficient. In addition to blood gas monitoring from the brachial artery during the operation, an oximeter probe was placed on the frontal area, and regional cerebral oximetry measurements were performed before and during the operation. The tests used in the study were determined and scored by the department of the neurology department. Verbal Memory Process Test (immediate memory score, learning score, and recall score) Wechsler Memory Scale (WMS) Digit Span, Verbal Fluency Test was used in the study. Neurocognitive tests of the patients undergoing carotid endarterectomy were performed at the outpatient clinic one day before the operation, on the postoperative $2 \mathrm{nd}$, 4th, and 30 th days. The test scores made before the operation were accepted as base points. The difference between the preoperative and postoperative points was examined.

Neurological tests were defined as follows:

1) Verbal Memory Process Test: There are 15 words in the first column, the words are said by the practitioner, and the patient repeats afterward. Each word known by the patient will be recorded as 1 point. The words he/she said wrong and repeated are determined by recording as -1 (minus one) point. This is recorded as an immediate memory score. The process is repeated a total of 10 times. The remembered words are recorded as a learning score. After 30 minutes, the first test is repeated and the words are asked to be remembered by the patient, which is recorded as recall score.

2) Wechsler Memory Scale (WMS) Digit Span: Forward span; starting with a 3-digit number, the patient is told one by one and asked to repeat. When successful, the number of digits is increased. If it is not successful, the second attempt is made with the same number of digits and a different number. The last number of digits that the patient is successful is determined as the forward digit range score. Backward span; the patient is asked to express the steps told to the patient in reverse. The scoring is the same as in the forward range.

3) Verbal Fluency Test: Within a minute, the patient is asked to say the animal names he knows. One point is awarded for each name. Names that are repeated more than once and that are irrelevant are recorded as -1 (minus one) points. The verbal fluency score is determined by summing up.

Inclusion criteria: Patients between the ages of 18-80, with no known CVO disease, a history of stroke, and no psychotic or neurotic disease, who read and signed the informed consent form, and underwent elective CEA operation were included. Because the evaluation of the patients with the given qualifications by neurocognitive tests would not be sufficiently leading to inaccurate statistical analysis.

Exclusion criteria: Those with a history of urgent operation, malignancy, a preoperative infection, preoperative kidney disease diagnosis (detected in any of preoperative laboratory tests), preoperative hepatic disease diagnosis (detected in any of preoperative laboratory tests), second operation for postoperative complications, history of stroke, and psychotic and neurotic disease, and who did not sign the informed consent form, were excluded from the evaluation.

Ethical statement: The design of the study was a retrospective study and approved by the Ethics Committee of Bozok University Medicine Faculty Hospital (Yozgat, Turkey) (2017-KAEK-189_2020.06.23_03).

Statistical analysis: Among descriptive statistics, frequency, percentage, mean, standard deviation, median, and minimum and maximum values are given. Normality assumptions were examined using the Shapiro-Wilk test. For the neurocognitive test scores, the differences in the preoperative, postoperative 2nd day, and postoperative 4th day and 30th day were evaluated by Friedman Test. When the result of the Friedman test was significant, paired comparisons were made with the Wilcoxon test. After the Bonferroni correction procedure, $\mathrm{P}$ values less than 0.05 were considered statistically significant. Analyses were made with SPSS 22.0 package program.

\section{RESULTS}

Education level of our cases: six (15.6\%) literate, 16 (42.1\%) primary school, eight $(21.1 \%)$ secondary school, five $(12.8 \%)$ high school, two (5\%) university, and one $(2.6 \%)$ doctorate level. (Table 1) Additional diseases of the cases are seen in Table 2. (Table 2) Thirty-two (84.21\%) of the cases were operated on under general anesthesia and six under local anesthesia. Eight of 18 cases with coronary artery disease had carotid and cardiac revascularization indications. Among these patients, priority was given to cardiac revascularization. In one case, combined surgery (carotid + coronary cardiopulmonary bypass) was performed. Of seven patients who underwent coronary bypass before CEA, off-pump coronary revascularization was achieved in four cases and on-pump coronary revascularization was achieved in three cases. Approximately 
Table 1. Education levels of the cases

\begin{tabular}{lcc}
\hline Education level & Rate (\%) & Number (n) \\
\hline Literate & $15.6 \%$ & 6 \\
Primary school & $42.1 \%$ & 16 \\
Secondary school & $21.1 \%$ & 8 \\
High school & $12.8 \%$ & 5 \\
University & $5 \%$ & 2 \\
Doctorate level & $2.6 \%$ & 1
\end{tabular}

Table 2. Additional diseases of the cases

\begin{tabular}{lcc}
\hline Additional disease & Rate (\%) & Number (n) \\
\hline Hypertension & $63.15 \%$ & 24 \\
Hyperlipidemia & $34.21 \%$ & 13 \\
Coronary artery disease & $47.36 \%$ & 18 \\
Diabetes mellitus & $52.63 \%$ & 20 \\
Cerebrovascular disease & $13.15 \%$ & 5 \\
Smoking habit & $39.47 \%$ & 15 \\
Peripheral arterial disease & $28.94 \%$ & 11 \\
COPD & $44.73 \%$ & 17 \\
Vitamin D insufficiency & $52.63 \%$ & 20 \\
Vitamin B12 insufficiency & $10.52 \%$ & 4 \\
\hline
\end{tabular}

COPD, chronic obstructive pulmonary disease

30 days after coronary revascularization, CEA was performed.

Left CEA was performed in 18 patients and right CEA in 20. The mean total operation time was $52.4 \pm 9$ minutes, the mean arterial clamping time was recorded as $21.8 \pm 3.2 \mathrm{~min}-$ utes. We used intravascular shunt in 19 patients with bilateral carotid disease for whom we predicted ischemia risk within the time of arterial clamping. Dacron was used in $21(55.26 \%)$ patients, PTFE in 12 patients $(31.57 \%)$, and saphenous vein in three patients $(8.57 \%)$ as the graft. Primary repair was done in two $(5.26 \%)$ patients. In the preoperative evaluation of the patients, although 20 of our patients $(52.63 \%)$ were followed up with a diagnosis of diabetes mellitus, seven $(18.42 \%)$ of these patients had uncontrolled blood glucose level regulation. Glycemia was regulated in hyperglycemic cases. In 13 (34.21\%) patients with hyperlipidemia, lipidemic control was achieved by regulating medical treatment in five $(13.15 \%)$ of the patients. Vitamin D deficiency was detected in 20 patients $(52.63 \%)$ and vitamin B12 deficiency in four patients $(10.52 \%)$. Vitamin deficiency was treated with substitution therapy in these patients and normalized levels were achieved. Five patients (14.28\%) presented to our clinic with a history of cerebrovascular disease (CVD) in the last three months. Cases with a fixed deficit, no deficit, and cases with infarction smaller than $2 \mathrm{~cm}$ on CT were operated after 15 days, and in other patients, CEA was performed at the end of the following 6-week recovery period. In angiography examination, unilateral carotid stenosis was present in $22(57.89 \%)$ cases, bilateral carotid stenosis in $16(42.10 \%)$ cases, ulcerated plaque in four $(10.52 \%)$ cases, and unilateral complete carotid stenosis in three $(7.89 \%)$ cases. After CEA, transient ischemic attack was observed in six $(15.78 \%)$ cases, dysphagia in three $(7.89 \%)$ patients, hypertension in nine $(23.68 \%)$ cases, hypotension in one $(2.63 \%)$ case, wound hematoma in four $(10.52 \%)$ patients, and wound infection in one case $(2.63 \%)$. With medical treatment, pathologies improved, and no mortality occurred. Neurocognitive tests were applied to our cases on the preoperative 1st day, and on the postoperative $2 \mathrm{nd}$, 4 th and 30 th days.

In the immediate memory score results, the mean value of the preoperative score was $5,10,3.90$ on the postoperative 2nd day, 3.94 on the postoperative 4th day, and 6.13 on the postoperative 30th day. According to these results, the biggest increase occurred on the postoperative 30th day, however, a statistically significant decrease was found between the preoperative values and postoperative 2 nd and 4th days. Although there is a significant decrease in the postoperative 4th day compared with the preoperative score and an increase compared with the postoperative 2 nd day, it is not statistically significant. A statistically significant increase in immediate memory score on the postoperative 30 th day compared with the preoperative and postoperative days 2 nd and 4th days were observed. (Table 3)

In the learning scores, according to preoperative scores, a decrease in the postoperative 2 nd and 4th days and an increase on the 30th day were found to be statistically significant. While the fall was apparent on the postoperative 2nd day, it increased less by being below the preop level on the postoperative 4th day and recovered on the postoperative 30th day. (Table 4)

In the recall scores, according to preoperative scores, a decrease on the postoperative 2 nd day and an increase on the postoperative 30 th day were found to be statistically significant. There was a statistically insignificant increase in the postoperative 4th day compared with the postoperative $2 \mathrm{nd}$ day. On the postoperative 30 th day, there was a significant increase compared with previous measurements. (Table 5)

Although the verbal fluency score results are better than the previous one in each measurement, the $P$-value is higher than 0.05 in the comparison of the measurements on the postoperative 2 nd and 4th days compared with the preoperative scores. It is not statistically significant. On the postoperative 30 th day, the $P$-value is less than 0.05 and is significant. While the increase between the postoperative 2 nd and 4 th days was insignificant, the increase between the postoperative 2 nd and 30th days, and the postoperative 4th and 30th days was found to be significant. According to these results, it can be claimed that verbal fluency improved significantly after one month following CEA. (Table 6) (Table 7)

WMS digit span scores: The increase on the postoperative 2 nd day compared with the preoperative scores are not statistically significant. The increase in the test score on the 
Table 3. Immediate memory score

\begin{tabular}{|c|c|c|c|c|c|c|}
\hline Preoperative immediate memory score & 5.10 & 2.34 & 5.00 & 2.00 & 12.00 & $<0.001 *$ \\
\hline Postoperative 4th day immediate memory score & 3.94 & 1.89 & 4.00 & 1.50 & 9.00 & Pre-post $4<0.001 *$ \\
\hline Postoperative 30th day immediate memory score & 6.13 & 2.29 & 6.00 & 3.00 & 12.00 & $<0.001 *$ \\
\hline
\end{tabular}

*Friedman Test, $P<0.05$; Min, minimum; Max, maximum

Table 4. Learning score

\begin{tabular}{|c|c|c|c|c|c|c|}
\hline Preoperative learning score & 46.57 & 8.90 & 45.5 & 32.00 & 64.00 & $<0.001 *$ \\
\hline Postoperative 4th day learning score & 45.97 & 8.40 & 47.0 & 30.00 & 64.00 & $<0.001 *$ \\
\hline Postoperative 30th day learning score & 50.47 & 7.80 & 50.0 & 35.00 & 66.00 & $<0.001 *$ \\
\hline
\end{tabular}

*Friedman Test, $P<0.05$; Min, minimum; Max: maximum

Table 5. Recall score

\begin{tabular}{|c|c|c|c|c|c|c|}
\hline & Mean & SD & Median & Min & Max & $P$ \\
\hline Preoperative recall score & 5.76 & 2.17 & 5.00 & 3.00 & 12.00 & $<0.001 *$ \\
\hline Postoperative 4th day recall score & 5.88 & 2.28 & 5.61 & 3.00 & 12.00 & 0.184 \\
\hline Postoperative 30th day recall score & 6.94 & 2.09 & 7.00 & 4.00 & 12.00 & $<0.001 *$ \\
\hline
\end{tabular}

*Friedman Test, $P<0.05$; Min, minimum; Max: maximum

Table 6. Verbal fluency score

\begin{tabular}{|c|c|c|c|c|c|c|}
\hline Preoperative verbal fluency score & 10.11 & 2.91 & 10.00 & 4 & 15 & 0.012 \\
\hline Postoperative 4th day verbal fluency score & 11.00 & 1.83 & 11.00 & 7 & 14 & $<0.001 *$ \\
\hline Postoperative 30th day verbal fluency score & 11.80 & 1.53 & 12.00 & 9 & 15 & $<0.001 *$ \\
\hline
\end{tabular}

*Friedman Test, $P<0.05$; Min, minimum; Max: maximum 
Table 7. Verbal fluency score comparison

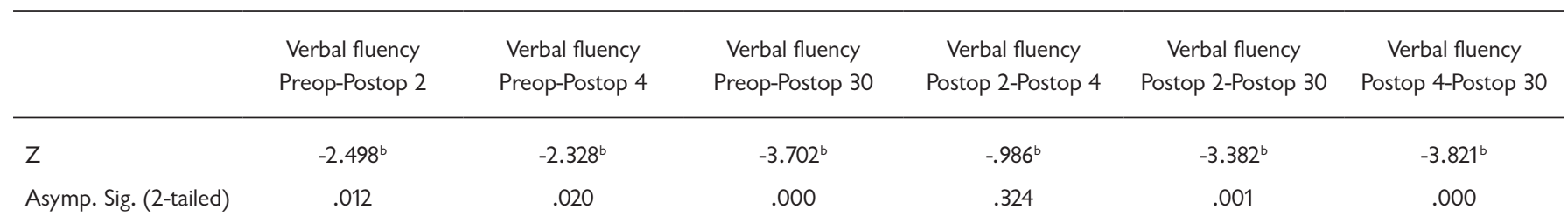

Table 8. Wechsler Memory Scale (WMS) Digit Span

\begin{tabular}{|c|c|c|c|c|c|c|}
\hline Preoperative WMS & 10.37 & 1.49 & 10.00 & 7 & 13 & $<0.001 *$ \\
\hline Postoperative 4th day WMS & 11.20 & 1.30 & 11.00 & 7 & 13 & $<0.001$ * \\
\hline Postoperative 30th day WMS & 11.54 & 0.98 & 12.00 & 9 & 13 & $<0.001 *$ \\
\hline
\end{tabular}

*Friedman Test, $P<0.05$; Min, minimum; Max: maximum

postoperative 4th and 30th days is significant compared with the preoperative scores. The increases between the postoperative 2 nd and 4th days and the postoperative 2 nd and 30 th days are significant, while the increase between the postoperative 4th and 30th days is not statistically significant. While evaluating the WMS digit span results, it can be stated that most of the improvement in the test score occurred on the 4th postoperative day. (Table 8 )

In four patients who had been detected to have vitamin B12 levels below $200 \mathrm{pg} / \mathrm{ml}$ in preoperative examinations, they were treated with $1000 \mu \mathrm{g}$ (microgram) every day for the first week, then $1000 \mu \mathrm{g}$ once a week for four weeks. When analyzed according to the average of the general group in this patient group, although there was no difference in the postoperative 2 nd and 4th days, it was determined that the mental score increase was $3 \%$ more than the general group at the end of the postop 1st month.

\section{DISCUSSION}

Various factors play a role in the formation of neurocognitive dysfunction, but ischemic and hemorrhagic causes also lead to a decrease in cognitive functions. In studies showing the relationship between carotid artery diseases and cognitive disorders, it has been reported that carotid artery stenosis leads to deterioration in cognitive abilities [Lal 2017]. In etiological studies, it has been shown that decreased cerebral perfusion is associated with cognitive impairment, which has shown that glutamate overstimulation and progressive neurodegeneration of neurons are a part of neuronal loss. VNCD is a progressive disease caused by a decrease in cerebral blood flow that causes a decrease in cognitive abilities [EthertonBeer 2014]. Microembolism caused by carotid vascular plaques and cerebral hemodynamic insufficiency is effective in the formation of VNCD [Lal 2017]. As a result, cerebral hypoperfusion and embolism should be avoided. CEA has been shown to prevent ischemic stroke due to carotid stenosis [European Carotid Surgery Trialists' Collaborative Group 1991]. Based on the aforementioned studies, surgical treatment of vascular insufficiency and unstable plaques is expected to improve cognitive functions as in stroke. However, studies are showing the opposite [Feliziani 2010; Rasmussen 2006]. Lunn et al. [Lunn 1999] and Irvine et al. [Irvine 1998] revealed the current lack of consensus regarding cognitive outcomes after CEA. In the literature review, 16 of 28 studies conducted on this subject show cognitive improvement after CEA. The remaining 12 studies did not show any improvement or decrease [Brajesh 2011]. In our results, there was a decrease in cognitive test scores in the early period after CEA, but significant increases in postoperative values in comparison to preoperative measurements were found, especially in the measurements one month after the surgery. The fact that carotid artery disease can produce cognitive impairment was first determined by Dr. Fisher, as based on an autopsy case in 1951 [Fisher 1951].

One of the most important factors affecting different results in neurocognitive abilities is the tests. There are many different tests used to measure neurocognitive abilities and a common methodology has not been established over time, and this is one of the factors that lead to conflicting results after CEA. In our study, we applied the verbal and quantitative tests recommended by the neurology department of our university one day before the surgery and on the postoperative 2nd, 4th, and 30th days. These tests are designed to detect changes in numerical, verbal abilities, and memory.

Anesthesia is one of the controversial applications in carotid surgery. In regional anesthesia, the patient's state of consciousness is instantly evaluated. Among the patients with a low-stress threshold, regional anesthesia may require 
a return to general anesthesia due to emotional and physical problems. Difficulties caused by urgent intubation at this stage may lead to additional complications. Although starting the operation directly with general anesthesia is safer for the physician and the patient against these existing reservations, it requires additional examinations for intraoperative monitoring. These examinations include A. cerebri media flow monitoring, transcranial Doppler, EEG, evoked potential measurements, carotid stump pressure, jugular venous oxygen pressure measurement, and cerebral oximetry. With these techniques, it is difficult to say which test is more specific and sensitive, considering that the comparative studies of the neurological conditions of postoperative patients with regional anesthesia are not prospective and randomized [Imparato 1993]. Our practice style is based on a patient-oriented approach. Clinical, psychological condition and patient preferences are taken into consideration when deciding on regional anesthesia. In our case series, general anesthesia was performed in 32 patients and regional anesthesia in six patients. We performed cerebral oximetry in cerebral monetarization in 32 cases undergoing general anesthesia. In the cases we started with regional anesthesia, we did not need to return to general anesthesia. We would like to draw attention to the importance of patient selection and the procedure training given to patients in preventing such complications by reducing the need to turn from regional anesthesia to general anesthesia.

There are different approaches in intraluminal shunt application to prevent actual ischemia in CEA surgeries. Some surgeons routinely use shunts under certain conditions, with or without monetarization, while others never use shunts under the same conditions [Girn 2008]. Arterial dissection, bleeding, thrombosis, or air embolism can be seen due to the use of shunts [Palombo 2007]. We think that it has a beneficial and risk-reducing effect in proper cases such as those with a history of previous CVD, impaired cerebral monetarization, carotid stump pressure below $40 \mathrm{mmHg}$, and contralateral $80 \%$ and above the obstruction. We used shunts in 19 patients. We did not encounter any complications, except one patient had a postoperative hematoma.

The coexistence rate of coronary artery and carotid artery diseases has been reported between $2.8 \%$ and $22 \%$. In this case, the treatment method to be applied is also among the controversial issues. Myocardial infarction is more common in patients who have undergone previous CEA. The risk of stroke is higher in those who have had CABC performed before [Illuminati 2011]. In 18 cases, a coexisting coronary artery disease was detected, and eight cases had an indication that required surgical treatment for both diseases. Coronary revascularization was achieved in eight patients. Carotid surgery was performed after an average of 30 days. In one case, combined surgery was performed.

Diabetes mellitus is associated with an increased risk for mild to moderate cognitive impairment [Biessels 2008]. The negative effect of chronic exposure to hyperglycemia on cognitive functions has been shown in diabetic patients [Brands 2005]. Early mean neurocognitive scores were found to be significantly better than the average overall score in patients with uncontrolled diabetes who had blood glucose regulation.
There was no significant difference between the mean general neurocognitive score and the postoperative 30th day. This result suggested that strict blood glucose regulation achieved by hospitalization led to significant improvements in neurocognitive scores.

Another controversial issue is patching in CEA. In the primary closure of the $5 \mathrm{~mm}$ diameter internal carotid artery, since the sutures on the edge of the arteriotomy will be $1 \mathrm{~mm}$ each, the vessel diameter will be $3 \mathrm{~mm}$ and the area will be 76\% [Archie 1998]. In such a case, the flow will significantly decrease. On the other hand, studies show that routine patch application has risks, such as prolonging the clamping time, double suture line, rupture, bleeding, infection, and thrombosis [Awad 1989]. In our clinical practice, we prefer to use a carotid patch in stenosis with a diameter of $5 \mathrm{~mm}$ or less and apply a primary closure technique in cases with a diameter of $6 \mathrm{~mm}$ and above. It has been suggested that the use of grafts is the right choice for those with internal carotid vessel diameter of less than $6 \mathrm{~mm}$, due to the intact flow dynamics and complications [Golledge 1996]. We used patches in 35 of 38 cases. We used Dacron in 21 cases, PTFE in 12 cases, and the saphenous vein in three cases. Primary closure was performed in three cases. We found no significant difference between patch, primary repair, and graft types, in terms of neurocognitive scores and complications.

Vitamin D induces prostacyclin in vascular smooth muscle cells, which prevents thrombus formation, cell adhesion, and smooth muscle cell proliferation [Wakasugi 1991]. The relationship between low vitamin D levels and endothelial dysfunction has been shown [Tarcin 2009]. In the preoperative examination of the cases, vitamin $\mathrm{D}$ hypovitaminosis was detected in 20 patients. Hypovitaminosis treatment was started as other comorbidities were treated before the operation. Studies are showing a close link between hypovitaminosis $\mathrm{D}$ and carotid atherosclerosis, based on 11 observational studies involving a total of 16,434 participants $[\mathrm{Fu}$ Hua Chen]. Carotid atherosclerosis is a mirror of the general atherosclerotic burden and the progression of subclinical atherosclerosis. Factors affecting atherosclerosis in vascular tissue are also effective in carotid arteries. We think that in the continuation of the mechanical treatment of carotid atherosclerosis with surgery, the treatment of glycemia, regulation of hyperlipidemia, and hypovitaminosis will contribute positively to endothelial healing.

Although Vitamin B12 deficiency is accepted as one of the causes of reversible dementia, it is still a subject of research on the relationship between losses of cognitive abilities [Oh 2003]. Studies showing the inverse correlation between Vitamin B12 levels and cognitive functions have been shown [Bell 1990]. Vitamin B12 deficiency also is known as a risk factor for vascular dementia and Alzheimer's disease [Clarke 1998]. In our cases, the use of substitute vitamin B12 treatment in addition to surgical treatment in patients with vitamin B12 deficiency in preoperative examinations, mental score increase was $3 \%$ more than the general group. Although the number of cases is low, this result makes us think that incomplete vitamin B12 replacement will contribute to the improvement in mental functions. 


\section{CONCLUSION}

In the past, CEA surgical treatment aimed to prevent ischemic stroke and CVD rather than neurocognitive recovery. Factors affecting neurocognition in CEA are multifactorial. Preservation and improvement of neurocognition are more important than any other period of history. By prioritizing cognitive abilities in the treatment of carotid stenosis, individualization of the treatment will help maximize the increase in cognitive abilities by providing optimum benefit to the patient of each factor.

Limitations: The main limitation of the study is the sample size calculation. This is an ongoing study and further results will be published in the future.

\section{REFERENCES}

Archie JP. 1998. Patching with Carotid arteriotomy: When to do it and What to use. Seminars in Vasc Surg. 11:24-9.

Awad JA, Little JR. 1989. Patch angioplasty in carotid endarterectomy: advantages, concerns, and controversies. Stroke. 20: 417-22.

Bell IR, Edman JS, Marby DW, Satlin A, Dreier T, Liptzin B, Cole JO. 1990. Vitamin B12 and folate status in acute geropsychiatric inpatients: affective and cognitive characteristics of a vitamin nondeficient population. Biol Psychiatry. 27: 125-137.

Biessels GJ, Deary IJ, Ryan CM. 2008. Cognition and diabetes: a lifespan perspective. Lancet Neurol. 7(2):184-90.

Bowler JV. 2002. The concept of vascular cognitive impairment. Journal of the Neurological Sciences. 203: 11-5.

Brajesh KL, Younes M, Cruz G, Kapadia I, Zafar J, Pappas PJ. 2011. Cognitive changes after surgery vs stenting for carotid artery stenosis. Journal of Vascular Surgery. Volume 54, Issue, Pages 691-698.

Brands AM, Biessels GJ, de Haan EH, Kappelle LJ, Kessels RP. 2005. The effects of type 1 diabetes on cognitive performance: a meta-analysis. Diabetes Care. 28(3):726-35.

Busse A, Bischkopf J, Riedel-Heller SG, Angermeyer MC. 2003. Mild cognitive impairment: prevalence and predictive validity according to current approaches Acta Neurol Scand. 108, pp. 71-81.

Clarke R, Smith AD, Jobst KA, Refsum H, Sutton L, Ueland PM. 1998. Folate, vitamin B12, and serum total homocysteine levels confirmed Alzheimer's disease. Arch Neurol. 55: 1449-1455.

Deiner S, Silverstein JH. 2009. Postoperative delirium and cognitive dysfunction. BJA. 103:141-6.

Etherton-Beer CD. 2014. Vascular cognitive impairment in dementia. Maturitas. 79:220-226.

European Carotid Surgery Trialists' Collaborative Group. 1991. MRC European Carotid Surgery Trial: interim results for symptomatic patients with severe (70-99\%) or with mild (0-29\%) carotid stenosis. Lancet. May 25;337:1235-43.

Feliziani FT, Polidori MC, De Rango P, et al. 2010. Cognitive performance in elderly patients undergoing carotid endarterectomy or carotid artery stenting: a twelve-month follow-up study. Cerebrovasc Dis. 30:244-51.

Fisher C. 1951. Senile dementia-A new explanation of its causation Arch
Neurol, 65, pp. 1-7.

Fu-Hua Chen BS, Tian Liu BS, Lei Xu MS, Lei Zhang MS, Xiao-Bo Zhou, MD. Association of Serum Vitamin D Level and Carotid Atherosclerosis: A Systematic Review and Meta-analysis.

Girn HR, Dellagrammaticas D, Laughlan K, Gough MJ. 2008. GALA Trial Collaborators. Carotid endarterectomy: technical practices of surgeons participating in the GALA trial. Eur J Vasc Endovasc Surg. 36(4):385-9.

Golledge J, Cuming R, Davies AH, Greenhalgh RM. 1996. The outcome of selective patching following carotid endarterectomy. Eur J Vasc Endovasc Surg. 11:458-63.

Hanning CD. 2005. Postoperative cognitive dysfunction. Br J Anaesth. 95:82-7.

Illuminati G, Ricco JB, Caliò F, Pacilè MA, Miraldi F, Frati G, et al. 2011. Short-term results of a randomized trial examining the timing of Carotid endarterectomy in patients with severe asymptomatic unilateral carotid stenosis undergoing coronary artery bypass grafting. J Vasc Surg. 54(4):993-9; discussion 998-9.

Imparato AM. 1993. Cerebral protection during anesthesia for Carotid and vertebral Surgery. In Bernstein EF, Collow AD, Nicolaides AN, et al. Cerebral revascularization London Med-Orion. p.405-505.

Irvine CD, Gardner FV, Davies AH, Lamont PM. 1998. Cognitive testing in patients undergoing carotid endarterectomy. Eur J Vasc Endovasc Surg. 15, pp. 195-204.

Jackson DC, Sandoval-Garcia C, Rocque BG, et al. 2015. Cognitive deficits in symptomatic and asymptomatic carotid endarterectomy surgical candidates. Archives of Clinical Neuropsychology. 31: 1-7.

Lal BK, Dux MC, Sikdar S, et al. 2017. Asymptomatic carotid stenosis is associated with cognitive impairment. Journal of vascular surgery. 66: 1083-92.

Lunn S, Crawley F, Harrison MJ, Brown MM, Newman SP. 1999. Impact of carotid endarterectomy upon cognitive functioning: A systematic review of the literatüre Cerebrovasc Dis. 9, pp. 74-81.

Mworozi K, Ameda F, Byanyima RK, and Nakasujja N. 2019. Carotid artery plaque detected on ultrasound is associated with the impaired $\operatorname{cog}$ nitive state in the elderly: A population-based study in Wakiso district, Uganda. Journal of Clinical Neuroscience.

North American Symptomatic Carotid Endarterectomy Trial Collaborators. 1991. The beneficial effect of carotid endarterectomy in symptomatic patients with high-grade symptomatic stenosis. N Engl J Med. 325: 445-53.

Oh R, Brown DL. 2003. Vitamin B12 deficiency. Am Fam Physician. 67: 979-986.

Palombo D, Lucertini G, Mambrini S, Zettin M. 2007. Subtle cerebral damage after shunting vs non-shunting during carotid endarterectomy. Eur J Vasc Endovasc Surg. 34(5):546-51.

Rasmussen LS. 2006. Postoperative cognitive dysfunction: incidence and prevention. Best Pract Res Clin Anaesthesiol. 20:315-30.

Tarcin O, Yavuz DG, Ozben B, Telli A, Ogunc AV, Yuksel M, et al. 2009. Effect of vitamin D deficiency and replacement on endothelial function in asymptomatic subjects. J Clin Endocrinol Metab. 94:4023-30.

Wakasugi M, Noguchi T, Inoue M, Kazama Y, Tawata M, Kanemaru Y, Onaya T. 1991. Vitamin D3 stimulates the production of prostacyclin by vascular smooth muscle cells. Prostaglandins. 42:127-136. 